\title{
miR-92b promotes autophagy and suppresses viability and invasion in breast cancer by targeting EZH2
}

\author{
FEI LIU ${ }^{1,2^{*}}$, MEIXIANG SANG ${ }^{1,2^{*}}$, LINGJIAO MENG ${ }^{1 *}$, LINA GU $^{1}$, \\ SHINA LIU ${ }^{1}$, JUAN LI ${ }^{1}$ and CUIZHI GENG ${ }^{3}$ \\ ${ }^{1}$ Research Center; ${ }^{2}$ Tumor Research Institute; ${ }^{3}$ Breast Center, \\ The Fourth Hospital of Hebei Medical University, Shijiazhuang, Hebei 050017, P.R. China
}

Received November 11, 2017; Accepted May 30, 2018

DOI: $10.3892 /$ ijo.2018.4486

\begin{abstract}
MicroRNAs (miRs) are a small non-coding RNA family with a length of 18-22 nucleotides. They are able to regulate gene expression by either triggering target messenger RNA degradation or by inhibiting mRNA translation. Enhancer of zeste homolog 2 (EZH2) is the core enzymatic subunit of polycomb repressor complex 2 and is responsible for the trimethylation of histone 3 on lysine 27 (H3K27me3); it is also able to silence a bundle of tumor suppressor genes through promoter binding. However, little is known regarding the effect of miR-92b on cell autophagy, viability and invasion as well as how it interacts with EZH2. The present study investigated the major role of miR-92b in the autophagy, viability and invasion of breast cancer. It was revealed that in MCF-7 and MDA-MB-453 cells, the expression of miR-92b promoted autophagy induced by starvation and rapamycin treatment. The results of in vitro experiments results demonstrated that miR-92b inhibited breast cancer cell viability, invasion and migration. To further elucidate the regulatory mechanisms of miR-92b in autophagy, a dual luciferase reporter assay was performed to determine whether miR-92b targeted the EZH2 gene. The expression of miR-92b was negatively correlated to EZH2 mRNA expression in breast cancer. Depletion of EZH2 induced phenocopied effects on miR-92b overexpression, thereby demonstrating its importance in autophagy. These results indicated that miR-92b may serve an important role in breast cancer in controlling autophagy, viability and invasion. The present study indicated that miR-92b and EZH2 may serve as potential biomarkers for cancer detection and highlighted their possible therapeutic implications.
\end{abstract}

Correspondence to: Professor Cuizhi Geng, Breast Center, The Fourth Hospital of Hebei Medical University, 12 Jiankang Road, Shijiazhuang, Hebei 050017, P.R. China

E-mail: cuizhigeng@hotmail.com

${ }^{*}$ Contributed equally

Key words: microRNA-92b, enhancer of zeste homolog 2, breast cancer, autophagy, viability, invasion

\section{Introduction}

Autophagy is the dynamic process in eukaryotes by which dysfunctional cytoplasmic components including damaged proteins and organelles are degraded in order to maintain neuron homeostasis $(1,2)$. It has been implicated in a number of physiological processes that are important for human health and disease. The central factors for regulating autophagy are adenosine monophosphate (AMP) kinase (AMPK) and mammalian target of rapamycin (mTOR). Nutrient depletion is one of the most effective physiological inducers of autophagy.

Limited energy in the form of adenosine triphosphate is able to active AMPK and drive autophagy. The kinase TOR (mTOR in mammals) exerts a negative effect on autophagy; the inhibition of mTOR activity by phosphorylating unc-51 like autophagy activating kinase $1 / 2$ (ULK1/2), as well as some autophagy-related (Atg) proteins, promotes autophagy (3). Autophagy serves an important role in cancer therapeutics. In precancerous lesions, a previous study indicated that autophagy enhancers may prevent the development of cancer (4). In advanced cancer, the inhibition and enhancement of autophagy have been suggested as potential treatment strategies (5-7).

In addition to intracellular signaling mechanisms, an increasing body of evidence has indicated that microRNAs (miRNAs/miRs) may serve a crucial role in the regulation of autophagy (8-10). miRNAs are a family of small non-coding RNA, with a length of 18-22 nucleotides, that regulate gene expression by either triggering target mRNA degradation or the inhibition of mRNA translation $(11,12)$. Multiple miRNAs has been shown to be able to regulate autophagy at various steps. For example, during the early stages of autophagy induction, miR-885-3p can directly modulate ULK2 expression upon cisplatin exposure (13). Beclin 1, a critical autophagypromoting gene, has been shown to serve a key role in the modulation of cell survival and death in different types of cells. Zhu et al (14) demonstrated that miR-30a regulates autophagy by inhibiting Beclin 1 expression. In addition, miR-375 directly suppressed autophagy by inhibiting the expression of ATG7 and impaired the viability of hepatocellular carcinoma cells upon hypoxic exposure (15). miR-376b has been observed to inhibit starvation- and rapamycin-induced autophagy in breast cancer cells by targeting the key autophagy proteins, Beclin 1 
and Atg4C (16). Furthermore, miR-181A blocked starvationand mTOR inhibition-associated autophagy by targeting the critical autophagy protein ATG5 (17). miR-638 has also been reported to directly target the tumor suppressor disheveled binding antagonist of $\beta$-catenin 3 and promote autophagy, as well as malignant phenotypes in cancer cells (18). In addition, some other miRNAs including miR-23a (19), miR-130a (20) and miR-124 (21) are also involved in the regulation of autophagy.

In recent years, an increasing body of evidence has indicated that miR-92b is dysregulated in various types of cancers (22). miR-92b promoted tumor migration, invasion and proliferation in osteosarcoma by targeting reversion inducing cysteine rich protein with Kazal motifs (23). It has also been reported to promote epithelial-mesenchymal transition in bladder cancer by targeting disabled homolog 2-interacting protein (24). These studies revealed that miR-92b may serve an oncogenic role in cancer. However, Zhao et al (25) reported that miR-92b targets mothers against decapentaplegic homolog 3 to inhibit the migration and invasion of nasopharyngeal cancer cells. These results demonstrated that one specific miRNA may serve various roles in distinct genetic contexts. To date, the potential function of miR-92b in breast cancer is still largely unknown.

In the present study, it was reported hsa-miR-92b promote starvation- and mTOR inhibition-induced autophagy, and inhibited the viability and invasion of breast cancer cells. Mechanistic studies revealed that miR-92b directly targeted the histone methyltransferase enhancer of zeste homolog 2 (EZH2), a crucial catalyzed subunit of Polycomb repressive complex 2 (PRC2), that regulates autophagy, proliferation and invasion in cancer cells. In addition, miR-92b promoted autophagy, and inhibited the viability and invasion of breast cancer cells by targeting EZH2. The results of the present study provided a novel strategy using miR- $92 b$ to regulate the progression of breast cancer.

\section{Materials and methods}

Plasmid constructs. miR-92b mimics, anti-miR-92b (anti-92b) and negative control (NC) RNA (miR-NC) were purchased from Shanghai GenePharma Co., Ltd. (Shanghai, China). Green fluorescent protein (GFP)-light chain 3 (LC3), EZH2 short hairpin (sh)-RNA Plasmid, pCMV6 Entry-EZH2Myc-DDK expression plasmid and control plasmid were purchased from OriGene Technologies, Inc. (Rockville, MD, USA). For the dual luciferase assay, the wild-type (wt) or mutant (mut) forms of the EZH2 3'-untranslated region (UTR) were cloned into the pGL3-promoter vector (Promega Corporation, Madison, WI, USA) using the following primers: EZH2 wt primers, 5'-GAATCTCGAGCATCTGCTAC-3' and 5'-CCGCTCGAGACAAGTTCAA-3'; EZH2 mut primers, 5'-ATGCAGTATGGTACATTTTTC-3' and 5'-AAAATTCA CTGGTACAAAAC-3'.

Cell culture. MCF-7 and MDA-MB-453 human breast carcinoma cells were cultured in Dulbecco's modified Eagle's medium (DMEM; Gibco; Thermo Fisher Scientific, Inc., Waltham, MA, USA) supplemented with $10 \%$ fetal bovine serum (FBS; Gibco; Thermo Fisher Scientific, Inc.) and penicillin/streptomycin (Gibco; Thermo Fisher Scientific, Inc.) in a 5\% $\mathrm{CO}_{2}$-humidified incubator at $37^{\circ} \mathrm{C}$. Starvation in Earle's balanced salt solution (EBSS; Biological Industries, Kibbutz Beit Haemek, Israel) at $37^{\circ} \mathrm{C}$ for $4 \mathrm{~h}$ or rapamycin treatment (2.5 $\mu \mathrm{M}$, at $37^{\circ} \mathrm{C}$ for $8 \mathrm{~h}$; BioVision, Inc., Milpitax, CA, USA) were used to induce autophagy.

Cell transfection. For miRNA transfection, MCF-7 and MDA-MB-453 cells were transfected with HiPerFect Transfection Reagent (Qiagen GmbH, Hilden, Germany). According to the manufacturer's instructions, $50 \mathrm{nM}$ of miR-92b mimics, anti-92b or miR-NC were added to each well of a 6-well plate. The sequences were as follows: miR-92b mimics, 5'-UAUUGCACUCGUCCCGGCCUCC-3' and 5'-AGGCCGGGACGAGUGCAAUAUU-3'; anti-92b, 5'-GGA GGCCGGGACGAGUGCAAUA-3'; and miR-NC, 5'-CAG UACUUUUGUGUAGUACAA-3'. For DNA transfection, $3 \mu \mathrm{g}$ plasmid DNA (GFP-LC3, EZH2 shRNA Plasmid, pCMV6 Entry-EZH2-Myc-DDK expression plasmid and control plasmid) was transfected with the FuGENE-HD transfection reagent (Promega Corporation) in each well of a 6-well plate. All subsequent cell experiments were performed at $24 \mathrm{~h}$ post-transfection.

GFP-LC3 analyses. For GFP-LC3 analysis, $48 \mathrm{~h}$ following GFP-LC3 and miRNA co-transfection, $2 \times 10^{6}$ cells were starved for $4 \mathrm{~h}$ in EBSS medium or treated with rapamycin $(2.5 \mu \mathrm{M})$ for $8 \mathrm{~h}$, fixed for $20 \mathrm{~min}$ in $4 \%$ formaldehyde at $37^{\circ} \mathrm{C}$, washed with PBS and then observed using a BX3-CBH fluorescence microscope (Olympus Corporation, Tokyo, Japan). Cells containing >10 GFP-LC3 green fluorescing dots were classed as positive. At least 150 GFP positive cells per condition were counted and the graphs were plotted as a percentage of GFP-LC3 positive cells over the total number of transfected cells.

Target gene prediction for miRNA. miRNA targets were identified using the bioinformatics software miRanda (www. microrna.org/microrna/home.do) and TargetScan Human version 7.1 (www.targetscan.org/vert_71/).

Western blot analysis. Total cellular proteins were harvested from cancer cell lines. Cancer cells were transfected with $50 \mathrm{nM}$ miR-92b, anti-92b, shEZH2 or miR-NC. Cell lysates were washed in ice-cold PBS and lysed in the radioimmunoprecipitation assay buffer $(0.1 \%$ SDS, $1 \%$ Triton, $0.5 \%$ deoxycholate, $0.5 \% \mathrm{NP} 40,50 \mathrm{mM}$ Tris- $\mathrm{HCl} \mathrm{pH} 7.5,150 \mathrm{mM} \mathrm{NaCl}, 50 \mathrm{mg} / \mathrm{ml}$ phenylmethylsulfonyl fluoride, $1 \mathrm{mg} / \mathrm{ml}$ leupeptin, $1 \mathrm{mg} / \mathrm{ml}$ aprotinin, $1 \mathrm{mg} / \mathrm{ml}$ pepstatin, $50 \mathrm{mg} / \mathrm{ml} \mathrm{Na}_{3} \mathrm{VO}_{4}$ and $50 \mathrm{mg} / \mathrm{ml}$ $\mathrm{NaF}$ ) with the complete mini protease inhibitor cocktail and PhosSTOP phosphatase inhibitor cocktail (Roche Applied Science, Penzberg, Germany). The protein concentration of cell lysates was determined by using the Pierce BCA Protein Assay kit (Thermo Fisher Scientific, Inc.) according to the manufacturer's instructions; $5 \%$ bovine serum albumin (BSA; Sigma-Aldrich; Merck KGaA, Darmstadt, Germany) was used as a standard. Equal amounts (30 $\mu \mathrm{g})$ of cell lysates were separated by $10 \%$ SDS-PAGE and transferred onto a polyvinylidene difluoride membrane (Bio-Rad Laboratories, Inc., Hercules, CA, USA). The membranes were blocked with 
TBS-T (50 mM Tris-HCI, pH 8.0, $100 \mathrm{mM} \mathrm{NaCl}$ and $0.1 \%$ Tween-20) containing 5\% non-fat dried milk or 5\% BSA (Sigma-Aldrich; Merck KGaA) for $1 \mathrm{~h}$ at room temperature, and then probed with the following primary antibodies overnight at $4^{\circ} \mathrm{C}$ : Rabbit-anti-human EZH2 antibodies (dilution, 1:1,000; cat. no. ab186006; Abcam, Cambridge, UK), rabbit-anti-human LC3B antibodies (dilution, 1:1,000; cat. no. ab192890; Abcam), rabbit-anti-human sequestosome 1 (SQSTM1)/p62 antibodies (dilution, 1:1,000; cat. no. GTX111393, GeneTex, Inc., Irvine, CA, USA) and rabbit-anti-human $\beta$-actin (dilution, 1:1,000; cat. no. 20536-1-AP; Wuhan Sanying Biotechnology, Wuhan, China). Following washing with TBS-Tween-20 for $15 \mathrm{~min}$, the membranes were incubated with horseradish peroxidaseconjugated secondary anti-rabbit antibodies (dilution, 1:1,000; cat. no. 7074s; Cell Signaling Technology, Inc., Danvers, MA, USA) for $1 \mathrm{~h}$ at room temperature. Finally, the immunoreactive bands were developed with Pierce Electro-Chemiluminescence Western blotting substrate (Thermo Fisher Scientific, Inc.) on Blue Basic autoradiography film (BioExpress Corporation, Kaysville, UT, USA). ImageJ software (National Institutes of Health, Bethesda, MD, USA) was employed to quantify protein levels.

Reverse transcription-quantitative polymerase chain reaction $(R T-q P C R)$. Extraction of total RNA, including miRNAs, from all culture cells was performed using the TRIzol reagent (Invitrogen; Thermo Fisher Scientific, Inc.) according to the manufacturer's instructions. Total RNA was then reverse transcribed into cDNA using the RevertAid First Strand cDNA Synthesis kit (Promega Corporation) with the following temperature protocol: $25^{\circ} \mathrm{C}$ for $5 \mathrm{~min}, 42^{\circ} \mathrm{C}$ for $60 \mathrm{~min}$ and $70^{\circ} \mathrm{C}$ for $15 \mathrm{~min}$. Human miRNAs were detected with their specific primers (Guangzhou RiboBio, Co., Ltd., Guangzhou, China). qPCR was performed using the SYBR Green Real-Time PCR Master Mix (Promega Corporation). The following primers were used for qPCR: hsa-miR-92b-3p forward, 5'-TATTGCACTCGTCCCGGCCTCC-3' and reverse, 5'-CAGTGCGTGTCGTGGAGT-3'; U6 forward, 5'-CTCGCT TCGGCAGCACA-3' and reverse, 5'-AACGCTTCA CGAATT TGCGT-3'; EZH2 forward, 5'-CGCTTTTCTGTAGGCGA TGT-3' and reverse, 5'-TGGGTGTTGCATGAAAAGAA-3'; GAPDH forward, 5'-CGCTGAGTACGTCGTGGAGTC-3' and reverse, 5'-GCTGATGATCTTGAGGCTGTTGTC-3'. The $2^{-\Delta \Delta C q}$ method (26) was used to evaluate the relative expression levels of the indicated genes.

Dual luciferase-reporter assay. pGL3-promoter vectors containing luc-EZH2-wt or luc-EZH2-mut were cotransfected using FuGENE-HD transfection reagent (Promega Corporation) in 6-well plates with miR-92b and Renilla luciferase into COS7 cells (The Cell Bank of Type Culture Collection of Chinese Academy of Sciences, Shanghai, China). Firefly and Renilla luciferase activities were measured $48 \mathrm{~h}$ following transfection using the dual-luciferase reporter assay system (Promega Corporation). The results of firefly luciferase activity were normalized to Renilla luciferase activity.

Transwell invasion and migration assays. Cell migration and invasion were investigated by Transwell assays. MCF7 and MDA-MB-453 cells were prepared following transfection with miR-NC, miR-92b or anti-92b for $24 \mathrm{~h}$, respectively, and the MCF7 cells were also prepared following transfection with vector, shEZH2, miR-NC, miR-92b, or co-transfection with miR-92b and EZH2 vector. Cells $\left(1 \times 10^{5}\right)$ were suspended in $200 \mu 1$ DMEM without FBS, were seeded in the upper chambers (Corning Incorporated, Corning, NY, USA). A total of $600 \mu \mathrm{l}$ DMEM with $10 \%$ FBS was added to the lower chambers. The chambers were then cultured at $37^{\circ} \mathrm{C}$ with $5 \% \mathrm{CO}_{2}$ for $24 \mathrm{~h}$. For invasion assays, Transwell chambers coated with Matrigel (BD Biosciences, Franklin Lakes, NJ, USA) were used. Cells on the upper chamber were then carefully removed, while the cells on the lower chamber were stained with crystal violet at $37^{\circ} \mathrm{C}$ for $10 \mathrm{~min}$. Cells were subsequently counted using a light microscope (magnification, $\mathrm{x} 200$ ) and five random fields of view were selected to calculate cell numbers.

Cell viability analyses. Cells were transfected with miRNAs (50 nM miR-92b mimics, anti-92b or miR-NC), and following $24 \mathrm{~h}$ post-transfection, cells were seeded into a 96-well plate $\left(3 \times 10^{3} /\right.$ well). Cell viability was measured using a Cell Counting Kit-8 (CCK8; Promega Corporation) at 24, 48 or $72 \mathrm{~h}$ following cell seeding. The viability of cancer cells was assessed by measuring the absorbance at $450 \mathrm{~nm}$ to obtain the optical density values.

Colony formation assay. A total of 3,000 cells were seeded per well in a 6-well cell culture plate, which were then transfected with $50 \mathrm{nM}$ miR-92b mimics, anti-92b or miR-NC, respectively, using HiPerFect Transfection Reagent. MDA-MB-453 cells were seeded in fresh 6-well plates and maintained in DMEM containing $10 \%$ FBS for 10 days. MCF7 cells were seeded on 6-well plates with 10\% FBS + DMEM for 2 weeks. Colonies were fixed with methanol at room temperature for $15 \mathrm{~min}$ and stained with crystal violet dye $(0.1 \% \mathrm{w} / \mathrm{v})$ for $15 \mathrm{~min}$ at room temperature.

Patients and tissue specimens. A total of 30 breast cancer tissues were collected from patients that underwent surgery between January 2015 and October 2016 at The Fourth Clinical Hospital of Hebei Medical University (Hebei, China). All of these patients were female, with a median age of 48 years, and did not receive preoperative adjuvant chemotherapy and radiotherapy. All tissues were placed in liquid nitrogen and stored at $-80^{\circ} \mathrm{C}$ following resection. The breast cancer tissues were then used to evaluate the expression of miR-92b and EZH2 via RT-qPCR, as aforementioned. The present study was approved by the Institutional Review Boards of The Fourth Clinical Hospital of Hebei Medical University and written informed consent was obtained from all patients.

Statistical analysis. A two-tailed Student's t-test was used to determine the differences between 2 groups. The associations among two or more variables was analyzed by one-way analysis of variance with Bonferroni's post hoc test. The correlation between miR-92b and EZH2 mRNA expression was examined by Spearman's correlation. $\mathrm{P}<0.05$ was considered to indicate a statistically significant difference. All analyses were performed with SPSS software (version 16.0; SPSS, Inc., Chicago, IL, USA) or GraphPad Prism (version 5; GraphPad Software, Inc., La Jolla, CA, USA). 
A
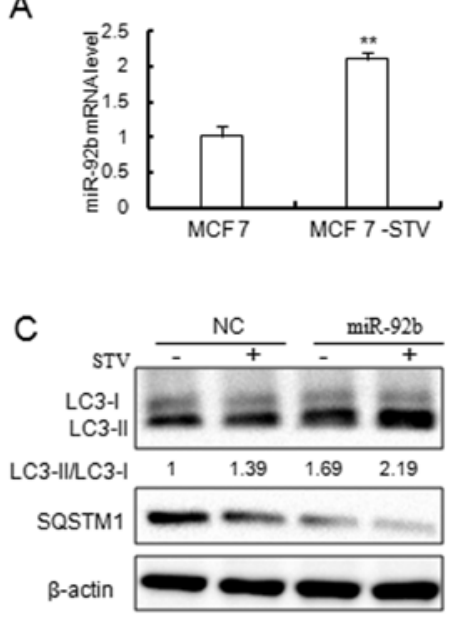

SQSTM1/ $\beta$-actin $1 \quad 0.7 \quad 0.44 \quad 0.2$

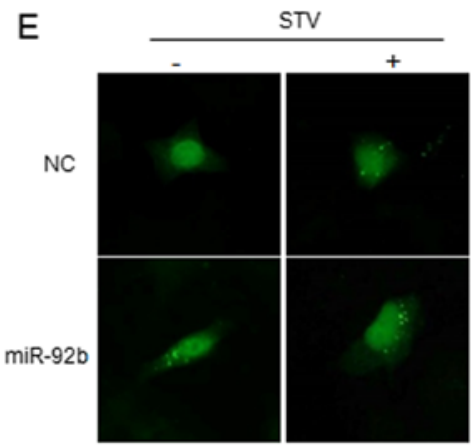

G

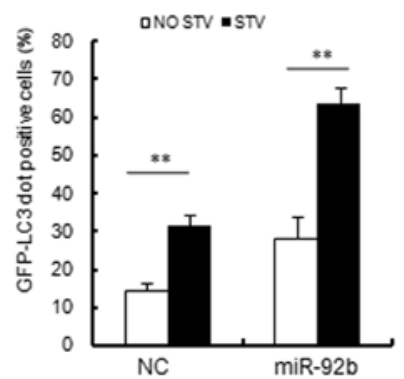

B

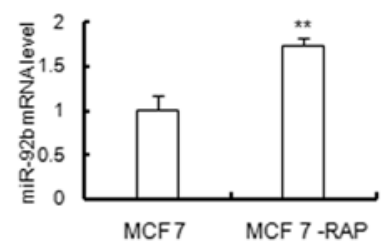

D

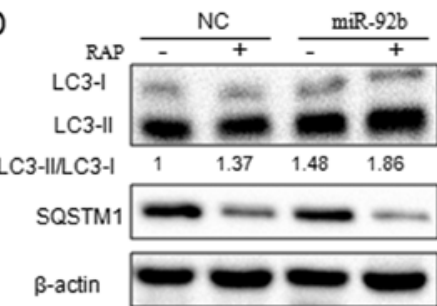

SQSTM1/ß-actin $1 \quad 0.38 \quad 0.67 \quad 0.24$

$\mathrm{F}$

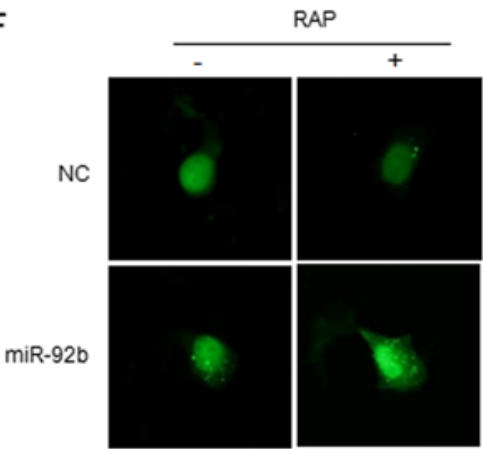

$\mathrm{H}$

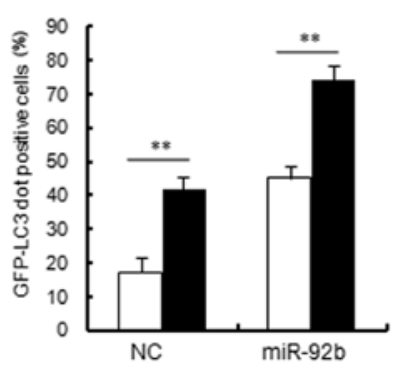

Figure 1. Overexpression of miR-92b increases autophagic activity in MCF-7 cells. (A and B) Reverse transcription-quantitative polymerase chain reaction analysis of miR-92b expression in MCF-7 cells treated with (A) STV (4 h) or (B) RAP (2.5 $\mu \mathrm{M}, 8 \mathrm{~h})$. miR-92b expression levels were significantly increased following STV or RAP treatment. ${ }^{* *} \mathrm{P}<0.01$ vs. MCF 7 cells. (C and D) Immunoblotting results indicated that miR-92b increased (C) STV and (D) RAP induced conversion of LC3-I to LC3-II and SQSTM1 degradation in MCF-7 cells. (E and F) miR-92b promoted STV and RAP induced GFP-LC3 dot formation in MCF-7 cells. Detection of cytoplasmic puncta formation by soluble GFP-fused LC3 protein is a commonly used method to follow autophagy activation using microscopy (magnification, $\mathrm{x} 40)$. ( $\mathrm{G}$ and $\mathrm{H})$ Quantitative analysis of the experiments in $(\mathrm{E}$ and $\mathrm{F}){ }^{* *} \mathrm{P}<0.01$, as indicated. STV, starvation; RAP, rapamycin; miR, microRNA; LC3, light chain 3; GFP, green fluorescent protein; SQSTM1, sequestosome 1; NC, negative control.

\section{Results}

miR-92b promotes starvation- or rapamycin-induced autophagy in MCF-7 cells. In order to detect whether miR-92b levels were enhanced following stress-inducing stimuli, the present study performed an RT-qPCR analysis. The results indicated that the miR-92b levels increased in MCF-7 cells that were starved for $4 \mathrm{~h}$ (Fig. 1A). In agreement with the starvation-associated results, treatment with rapamycin also elevated miR-92b expression in MCF-7 cells (Fig. 1B).

Further study revealed that miR-92b enhanced autophagy following starvation in MCF-7 cells (Fig. 1C-H). As shown in
Fig. 1C, overexpression of miR-92b increased the transformation of free LC3-I to the autophagy membrane associated LC3-II, which was induced by starvation. In addition, miR-92b was markedly associated with starvation-induced SQSTM1 degradation (Fig. 1C). Overexpression of miR-92b also increased the accumulation of starvation-induced GFP-LC3 dots (Fig. 1E and G). These results indicated that miR-92b may serve a role in regulating starvation-induced autophagy.

Similarly, the present study evaluated whether miR-92b overexpression also affects autophagy induced by mTOR-inhibitor rapamycin. Consistent with the results of starvation, rapamycin induced LC3 lipidation (Fig. 1D), SQSTM1 degra- 
A
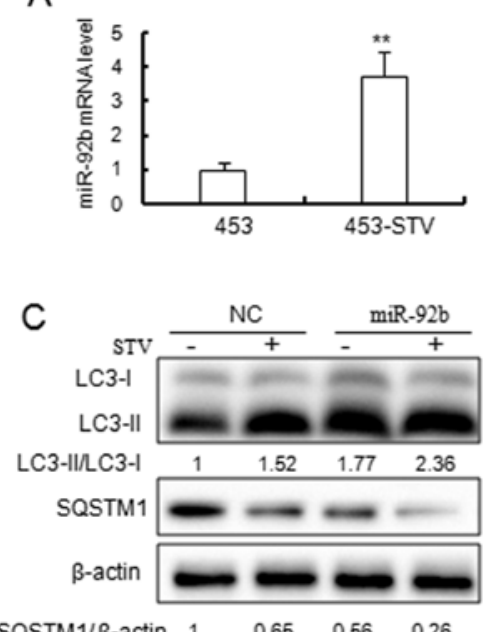

E

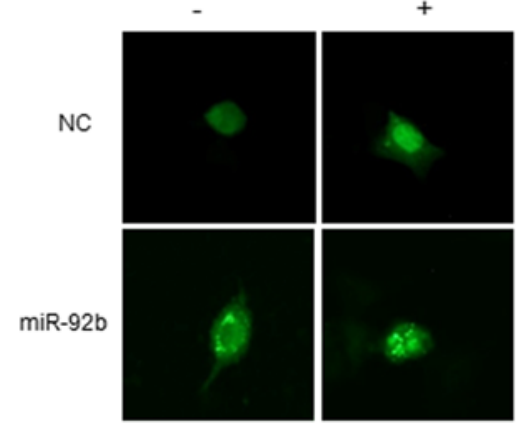

G

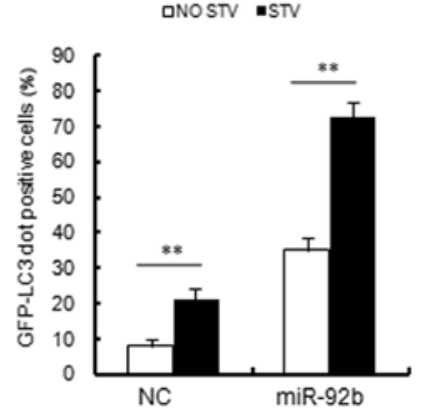

B

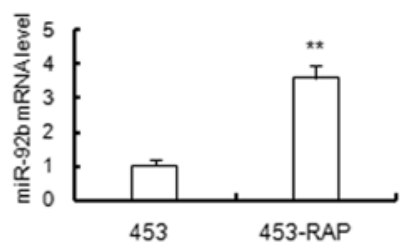

D
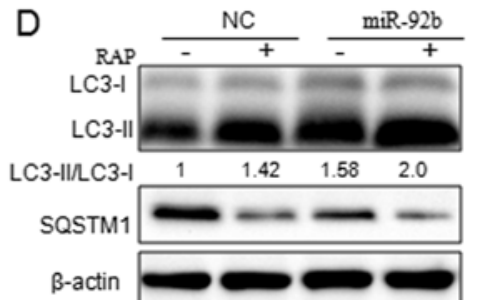

SQSTM1/ß-actin $1 \quad 0.44 \quad 0.60 \quad 0.22$

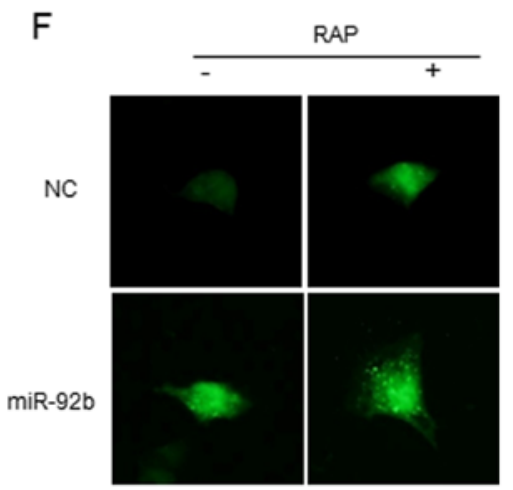

$\mathrm{H}$

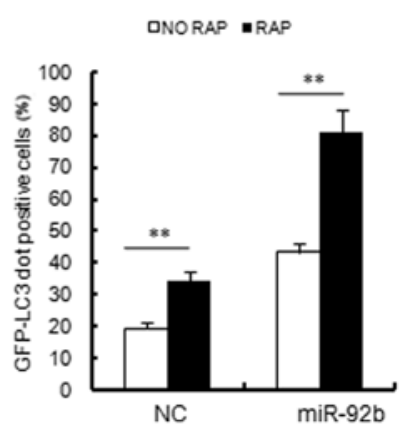

Figure 2. Overexpression of miR-92b results in increased autophagic flux in MDA-MB-453 cells. (A) STV (4 h) and (B) RAP (2.5 $\mu \mathrm{M}, 8 \mathrm{~h})$ induced miR-92b expression in MDA-MB-453 cells. miR-92b expression was detected by reverse transcription-quantitative polymerase chain reaction. ${ }^{* *} \mathrm{P}<0.01 \mathrm{vs} .453$ cells. (C and D) Immunoblotting results indicated that miR-92b increased (C) STV and (D) RAP induced conversion of LC3-I to LC3-II and SQSTM1 degradation in MDA-MB-453 cells. (E and F) miR-92b promoted (E) STV and (F) RAP induced GFP-LC3 dot formation in MDA-MB-453 cells (magnification, x40). $(\mathrm{G}$ and $\mathrm{H})$ Quantitative analysis of the experiments in $(\mathrm{E}$ and $\mathrm{F}) .{ }^{* *} \mathrm{P}<0.01$, as indicated. STV, starvation; RAP, rapamycin; miR, microRNA; LC3, light chain 3; GFP, green fluorescent protein; SQSTM1, sequestosome 1; NC, negative control; 453, MDA-MB-453 cells.

dation (Fig. 1D) and GFP-LC3 dot formation (Fig. 1F and H). Therefore, in addition to its effect on autophagy induced by starvation, miR-92b also promoted the autophagic activity induced by rapamycin in MCF-7 cells.

miR-92b promotes starvation- or rapamycin-induced autophagy in MDA-MB-453 cells. In order to confirm the effect of miR-92b on autophagy in breast cancer cells, the present study selected MDA-MB-453 cells, which have the same low expression of miR-92b as MCF7 cells. Starvation and treatment with the mTOR-inhibitor rapamycin increased endogenous miR-92b expression (Fig. 2A and B). Similarly, the overexpression of miR-92b markedly increased the lipidation of LC3 and SQSTM1 degradation (Fig. 2C and D) in MDA-MB-453 cells. It was further observed that autophagosome accumulation was enhanced in MDA-MB-453 cells overexpressing miR-92b (Fig. 2E-H).

miR-92b inhibits the invasion and migration ability of breast cancer cells. In order to examine the effect of miR-92b on the malignant biological processes in breast cancer cells, the present study firstly examined the role of miR-92b in 
A
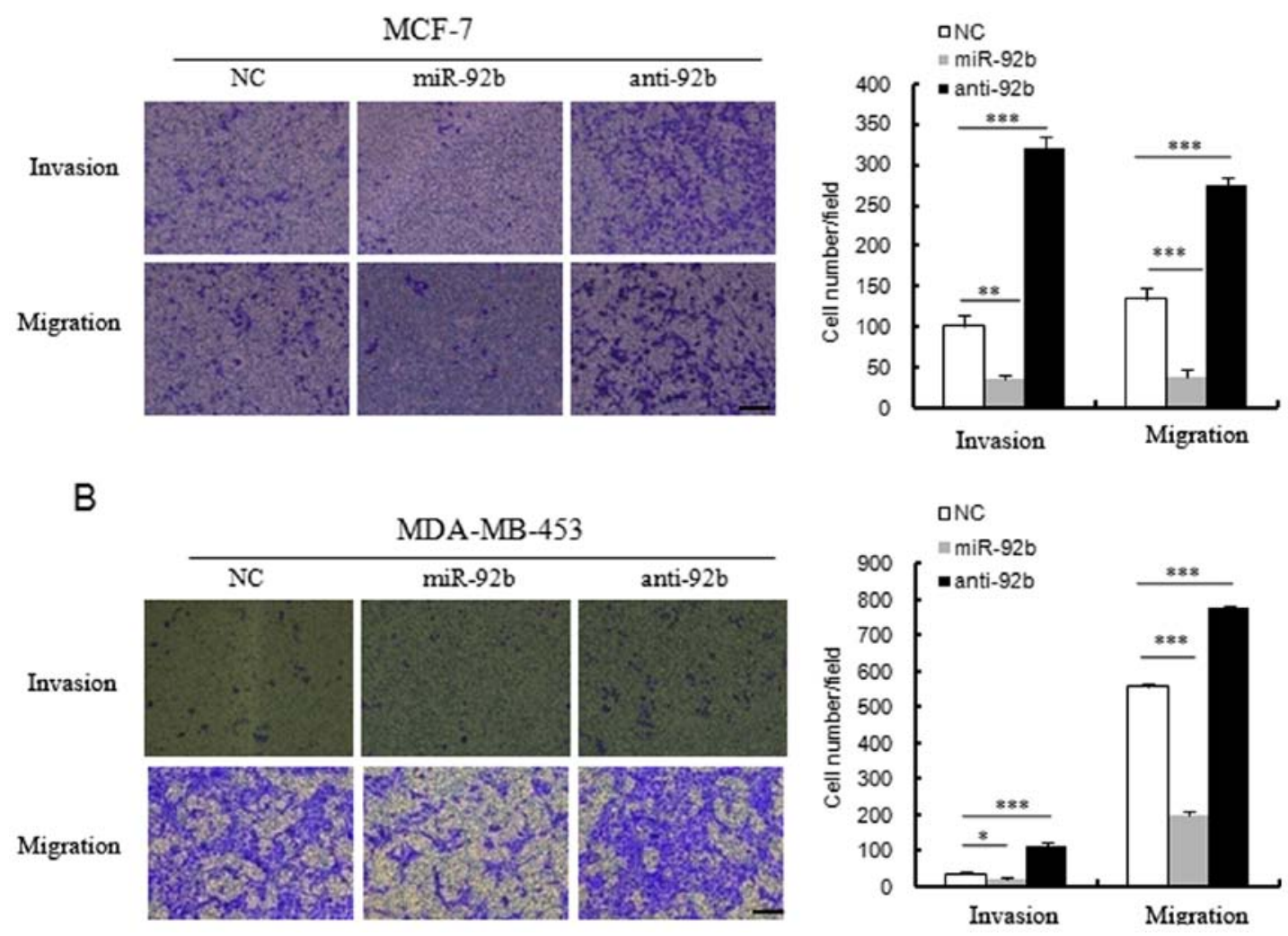

Figure 3. Effects of miR-92b on breast cancer cell migration and invasion. (A) miR-92b suppressed the migration and invasion of MCF-7 cells. However, silencing miR-92b expression promoted migration and invasion. (B) miR-92b promotes the migration and invasion ability of MDA-MB-453 cells. Scale bar $=100 \mu \mathrm{m}$; magnification, $\mathrm{x} 200) .{ }^{*} \mathrm{P}<0.05,{ }^{* *} \mathrm{P}<0.01$ and ${ }^{* * * *} \mathrm{P}<0.001$, as indicated. miR, microRNA; NC, negative control.

the migration of MCF7 and MDA-MB-453 cells. Transwell migration assays indicated that the number of migrating cells was reduced in miR-92b mimics-transfected MCF7 and MDA-MB-453 cells when compared with the control group (Fig. 3A and B). In addition, the suppression of endogenous miR-92b with its inhibitor enhanced cell motility when compared with control cells (Fig. 3A and B).

Furthermore, the Matrigel invasion assay system was used to examine the effect of miR-92b on MCF7 and MDA-MB-453 cell invasion ability. Overexpression of miR-92b decreased the invasion ability of MCF7 and MDA-MB-453 cells (Fig. 3A and B). Consistently, transfection with the miR-92b inhibitor resulted in the enhanced invasion ability of these cells (Fig. 3A and B). Therefore, these results demonstrated that miR-92b can affect the migration and invasion ability of breast cancer cells.

miR-92b inhibits the viability of breast cancer cells. In order to examine the effects of miR-92b on breast cancer cell viability, the present study performed CCK8 assays and colony formation assays. When compared with the control groups, the transfection-induced overexpression of miR-92b in breast cancer cells suppressed the viability of breast cancer cells over time (Fig. 4A and B). By contrast, suppressing endogenous miR-92b with its inhibitor promoted the viability of breast cancer cells (Fig. 4A and B).

In line with the cell viability assays, the colony formation assays also demonstrated that the MCF7 and MDA-MB-453 cells transfected with miR-92b exhibited decreased colony formation abilities in comparison with those of the control groups (Fig. 4C and D). In addition, suppressing endogenous miR-92b with its inhibitor enhanced colony formation (Fig. 4C and D).

$E Z H 2$ is a direct target of $m i R-92 b$. TargetScan analysis revealed that there was a miR-92b binding site in the 3'-UTR region of EZH2 (Fig. 5A). In order to eliminate the individual differences caused by transfection efficiency issues, the present study chose to use cos7 cells for the Luciferase reporter assay due to their high transfection efficiency. The luciferase reporter assay demonstrated that luciferase activity was inhibited by co-transfection the wild-type 3'-UTR of EZH2 and miR-92b mimic, and the deletion of the miR-92b target site inhibited this decrease in luciferase activity (Fig. 5B).

Then, the expression of miR-92b and EZH 2 mRNA in 30 pairs of breast cancer tissues was analyzed by RT-qPCR. Similarly, a negative correlation was observed between miR-92b expression and EZH2 mRNA expression in breast cancer specimens $(\mathrm{P}<0.001$; Fig. 5C).

In addition, the present study also examined EZH2 protein expression levels in MCF7 cells transfected with miR-92b mimics, miR-92b inhibitor or miR-NC (Fig. 5D and E). The EZH2 protein levels were reduced in MCF7 cells with miR-92b overexpression (Fig. 5D). In line with this, EZH2 protein expression was upregulated in miR-92b inhibitor transfected MCF7 cells (Fig. 5E). 
A

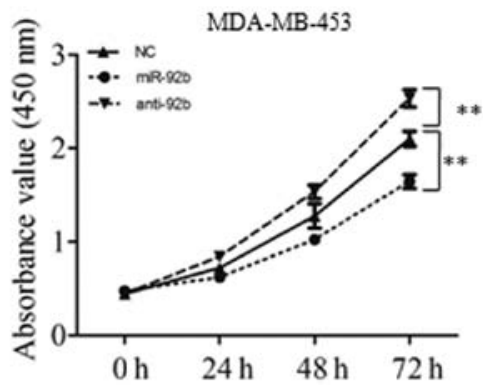

C

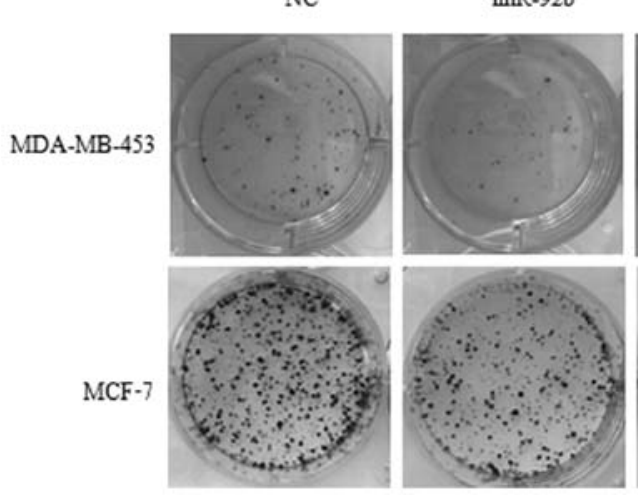

B

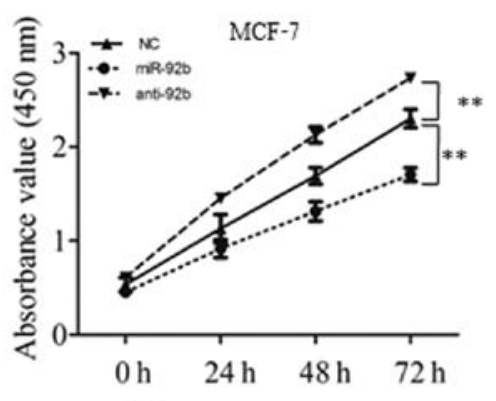

D
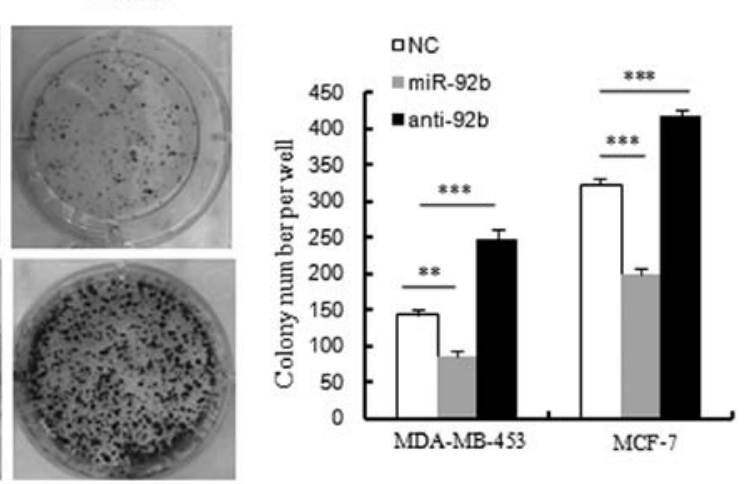

Figure 4. miR-92b inhibits cancer cell proliferation. (A and B) Overexpression of miR-92b inhibited cancer cells growth. However, silencing miR-92b expression promoted cell proliferation in (A) MDA-MB-453 and (B) MCF-7 cells. The cell viability was measured from 0 to $72 \mathrm{~h}$ by Cell Counting Kit- 8 assay. (C and D) Colony formation assays transfecting MCF-7 and MDA-MB-453 cells with miR-92b mimics or inhibitor. ${ }^{* *} \mathrm{P}<0.01$ and ${ }^{* * * *} \mathrm{P}<0.001$, as indicated. miR, microRNA; NC, negative control.

A

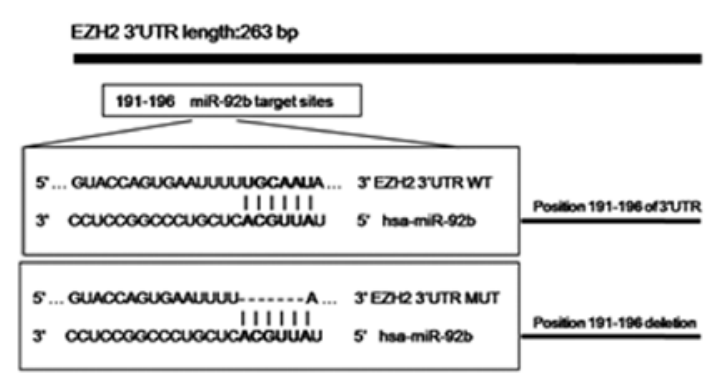

C

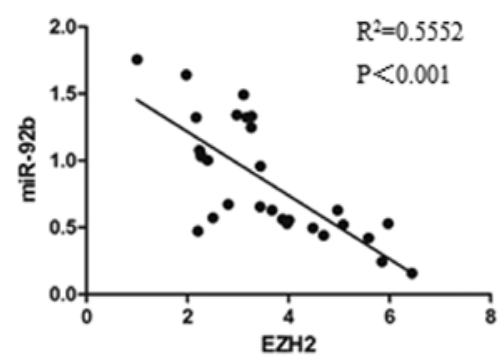

B

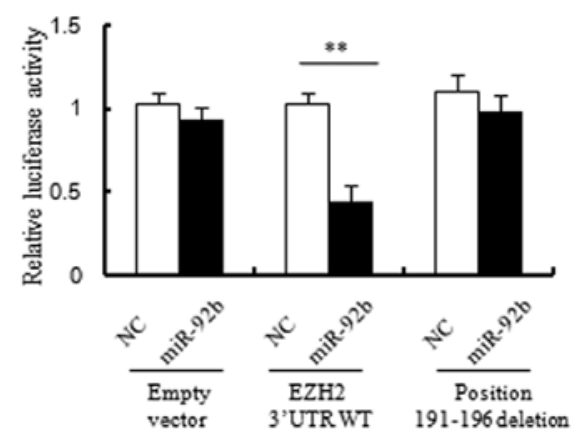

D

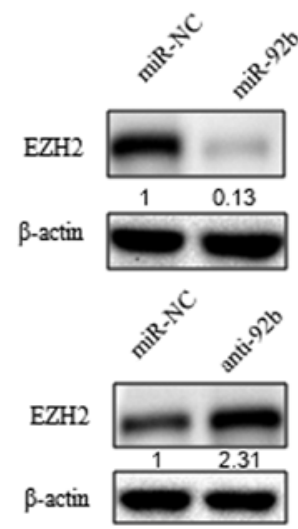

Figure 5. Prediction and validation of EZH2 as a direct target of miR-92b. (A) Schematic of the predicted binding site of miR-92b in EZH2 3'-UTR. A mutant EZH2 3'-UTR construct was tested in parallel. (B) The wild-type or mutant forms of EZH2 3'-UTR were co-transfected into COS7 cells with miR-92b mimic or NC RNA. Luciferase activity was measured at $48 \mathrm{~h}$ post-transfection. The results were normalized against Renilla luciferase values. ${ }^{* *} \mathrm{P}<0.01$, as indicated. (C) Negative correlation between miR-92b and EZH2 mRNA levels in breast specimens. (D) EZH2 protein expression was reduced in MCF7 cells with miR-92b overexpression in MCF7 cells. (E) EZH2 protein expression was reduced in miR-92b mimics transfected MCF7 cells. EZH2, enhancer of zeste homolog 2; miR, microRNA; STV, starvation; NC, negative control; UTR, untranslated region; WT, wild-type; MUT, mutant. 

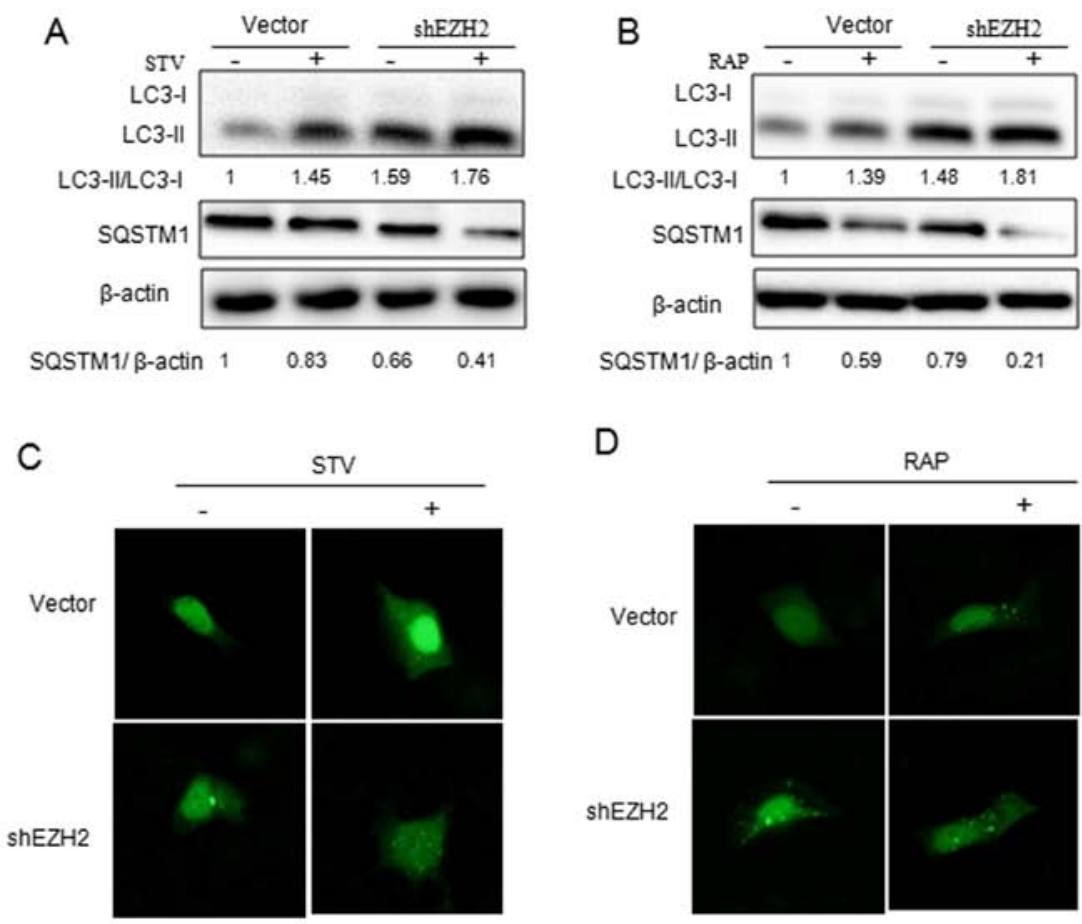

E

F
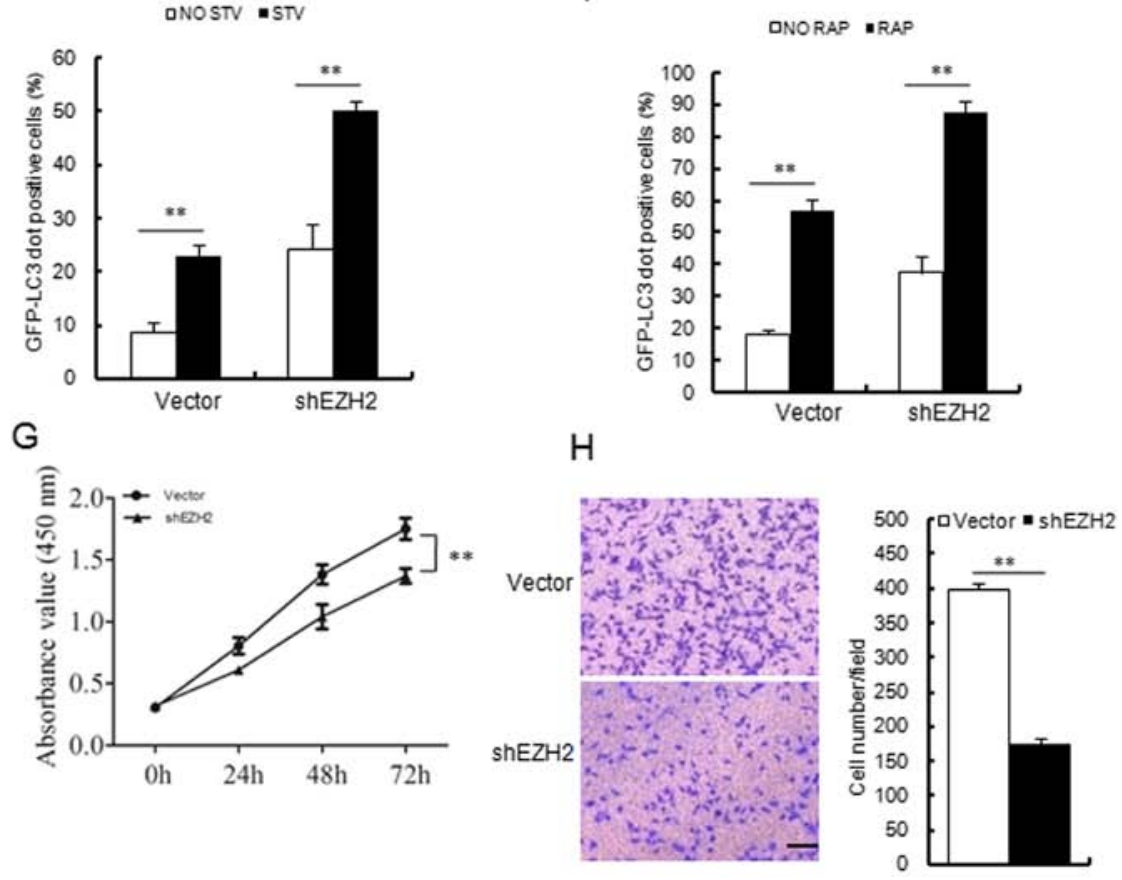

Figure 6. Silencing EZH2 expression with EZH2 shRNA promotes autophagy, and suppresses proliferation and invasion in MCF-7 cells. (A and B) Immunoblotting results indicated that shEZH2 increased (A) STV and (B) RAP induced conversion of LC3-I to LC3-II and SQSTM1 degradation. (C and D) shEZH2 promoted STV and RAP induced GFP-LC3 dot formation in MCF-7 cells (magnification, x40). (E and F) Quantitative analyses of the experiments in panels (C and D). $(\mathrm{G}$ and $\mathrm{H})$ shEZH2 inhibited the proliferation and invasion abilities of MCF-7 cells. Scale bars $=100 \mu \mathrm{m}$; magnification, $\mathrm{x} 200 .{ }^{* *} \mathrm{P}<0.01$, as indicated. EZH2, enhancer of zeste homolog 2; STV, starvation; RAP, rapamycin; miR, microRNA; shRNA, short hairpin RNA; LC3, light chain 3; GFP, green fluorescent protein; SQSTM1, sequestosome 1; NC, negative control.

Effects of EZH2 silencing on autophagy, viability and invasion. To evaluate the effects of EZH2 on autophagy, the present study transfected MCF7 cells with EZH2 shRNA or Empty vector and analyzed the levels of autophagy induced by starvation or rapamycin treatment. As shown in Fig. 6, EZH2 silencing enhanced the autophagy of MCF-7 cells. EZH2 shRNAs enhanced the lipidation of LC3 and SQSTM1 degradation (Fig. 6A and B) induced by starvation or rapamycin.
Similarly, EZH2 silencing also enhanced starvation- or rapamycin-induced GFP-LC3 dot accumulation (Fig. 6C-F).

The present study then examined the effects of EZH2 knockdown on breast cancer cell growth and invasion. shEZH2 plasmid and the control empty plasmid were transfected into MCF-7 cells. According to the results presented in Fig. 6G, cell viability was inhibited following shEZH2 plasmid transfection. In addition, the invasion ability of MCF7 cells was 
A

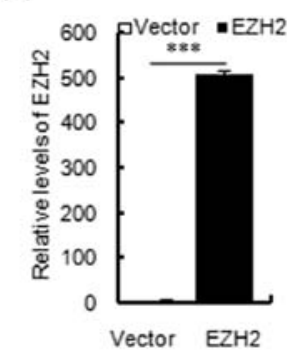

B

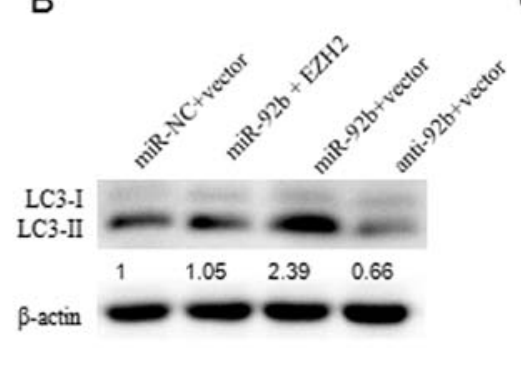

C
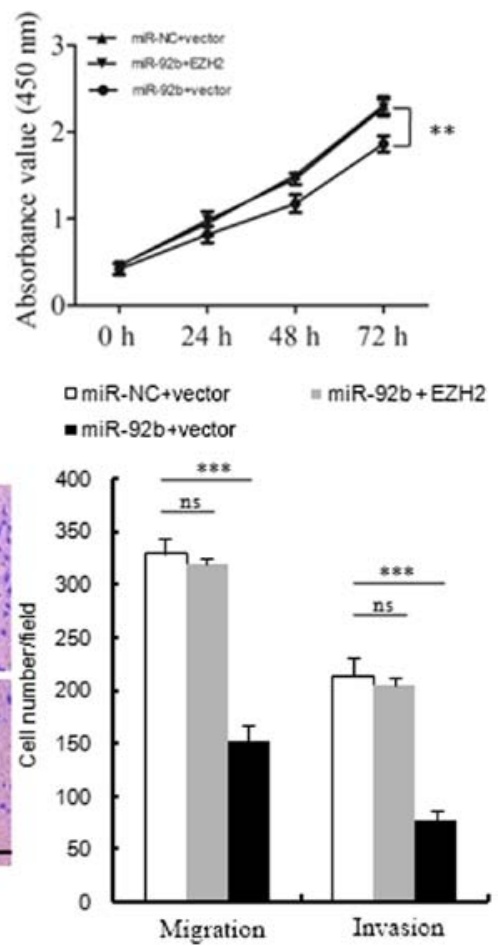

Figure 7. EZH2 is involved in miR-92b regulated cell autophagy, proliferation and invasion. (A) Reverse transcription-quantitative polymerase chain reaction revealed the relative mRNA expression of EZH2 in MCF7 cells transfected with the pCMV6 Entry-EZH2-Myc-DDK expression plasmid or the control plasmid. (B) Western blot analyses of LC3 expression in MCF-7 cells transfected with miR-NC plus vector, miR-92b plus EZH2, miR-92b plus vector or antimiR-92b (anti-92b) plus vector. Autophagy-associated LC3-I to LC3-II conversion (LC3 lipidation) was attenuated in anti-miR-92b plus vector overexpressing cell extracts, when compared with miR-NC plus vector as the control group. (C) Cell Counting Kit-8 analysis of MCF7 cells transfected with miR-NC plus vector, miR-92b plus EZH2 and miR-92b plus vector at the indicated times. (D) Transwell analysis of MCF-7 cells treated with miR-NC plus vector, miR-92b plus EZH2 and miR-92b plus vector, and the quantitative analysis is shown in the right-hand panel. Scale bar $=100 \mu \mathrm{m} ;$ magnification, $\mathrm{x} 200$. ${ }^{* *} \mathrm{P}<0.01$ and ${ }^{* * * *} \mathrm{P}<0.001$, as indicated. EZH2, enhancer of zeste homolog 2; miR, microRNA; LC3, light chain 3; NC, negative control; ns, not significant.

also inhibited in the shEZH2 plasmid transfected group when compared with the vector control group (Fig. $6 \mathrm{H}$ ).

EZH2 is involved in miR-92b regulated cell autophagy, viability, migration and invasion. To further verify the functional connection between miR-92b and EZH2, MCF7 cells were cotransfected with miR-92b mimic and EZH2 expression vector or control (Fig. 7A). Overexpression of EZH2 was demonstrated to reverse the inhibitory effects of miR-92b on cell autophagy, viability, migration and invasion in MCF7 cells (Fig. 7B-D). These results indicated that miR-92b may inhibit cell autophagy, viability and invasion by regulating EZH2 expression.

\section{Discussion}

In the present study, it was revealed that endogenous miR-92b expression levels are elevated in response to different autophagy inducing stimuli. Notably, miR-92b overexpression enhanced GFP-LC3 dot formation, the lipidation of LC3 and SQSTM1 degradation. These results were observed following starvation and rapamycin treatment in MCF7 and MDA-MB-453 cells. Additionally, it was detected that overexpression of miR-92b in MCF7 and MDA-MB-453 cells decreased tumor cell viability, migration and invasion. The present study also identified a novel miR-92b target, EZH2, and demonstrated that shRNA-mediated knockdown of EZH2 phenocopied the effect of miR-92b, suggesting that miR-92b may exert its pro- autophagic function via EZH2. Taken together, these results indicated that miR-92b may negatively regulate the expression of EZH2, promoting autophagy, and decreasing tumor cell viability, migration and invasion.

miR-92b is involved in the progression of the central neural system development and the development of several tumors $(27,28)$. Previous reports on glioblastoma and nonsmall cell lung cancer have suggested that miR-92b promoted neoplastic cell growth and metastasis $(29,30)$. However, in esophageal squamous cell carcinoma, miR-92b did not stimulate apoptosis or inhibit proliferation, though it did impair the motility of cancer cells $(31,32)$. In accordance with Ma et al $(31,32)$, the present study observed that there was significantly reduced miR-92b levels in human breast cancer tissues in comparison with normal tissue specimens, and overexpression of miR-92b in breast cancer cells decreased tumor cell proliferation, invasion and migration. These results supported the notion that one specific miRNA may exert a variety of effects in different genetic contexts (33). The mechanism for miR-92b underlying a tumors potential is rather complex, as it is mediated by a varied range of targets that are potentially variable depending on the type of cancer and the environmental stimuli. The results of the present study provide further support for the functional relevance of miR-92b in breast cancer via the identification of the methyltransferase EZH2 as its novel target during starvation- and rapamycininduced autophagy. 
$\mathrm{EZH} 2$ is the core catalytic subunit of the PRC2 complex. PRC2 methylates the lysine residue at position 27 of histone 3 (H3K27) $(34,35)$, which results in chromatin compaction and epigenetic silencing of genes. EZH2 overexpression has been reported in different types of malignancies, and was associated with malignant tumor growth, metastasis and poor prognosis in a variety of human malignancies (36-39). Recently, it has been reported that EZH2 may serve an important role in autophagy (40). Notably, the present study demonstrated the post-transcriptional modification of EZH2 by miR-92b and its involvement in autophagy. It has been indicated that EZH2-mediated gene promoter methylation can promote the malignant phenotype of different cancer cells, due to the loss of tumor suppressor gene functions, which results in the transformation of stem cells into cancer cells (41-43); this may partly explain the reduced malignant properties induced by miR-92b.

In conclusion, the present study confirmed that miR-92b may be a crucial regulator of starvation- and rapamycininduced autophagy by targeting the methyltransferase EZH2. This post-transcriptional regulation had a significant effect on inhibiting the oncogenic phenotypes of tumor cells. In view of these results revealing enhanced miR-92b expression and attenuated EZH2 expression in human breast cancer cells, it was hypothesized that the autophagy-associated miR-92b-EZH2 axis may be an appealing target for therapeutic interventions in breast cancer.

\section{Acknowledgements}

Not applicable.

\section{Funding}

The present study was supported by the Medical Science Research Foundation of Health and Family Planning Commission of Hebei (grant no. 20180532), the Science Fund for Outstanding Youth of Hebei Province (grant no. 2016206410) and the Science and Technology Supporting Program of Hebei Province (grant no. 152777184).

\section{Availability of data and materials}

All data generated or analyzed during this study are included in this published article.

\section{Authors' contributions}

FL, MS and LM performed the molecular studies and drafted the manuscript. SL and JL collected the clinical data and tissue samples. FL and LG performed the statistical analysis. CG designed the study and helped to draft the manuscript. All authors read and approved the final manuscript.

\section{Ethics approval and consent to participate}

The use of human tissues was approved by the Ethics Committee of the Fourth Hospital of Hebei Medical University (Hebei, China) and written informed consent was obtained from all patients.

\section{Patient consent for publication}

Consent for the publication of the clinical and pathological data was obtained from all patients who were involved in the present study.

\section{Competing interests}

The authors declare that they have no competing interests.

\section{References}

1. Mizushima N: Autophagy: Process and function. Genes Dev 21: 2861-2873, 2007.

2. Mizushima N, Yoshimori T and Ohsumi Y: The role of Atg proteins in autophagosome formation. Annu Rev Cell Dev Biol 27: 107-132, 2011.

3. Klionsky DJ: Autophagy: From phenomenology to molecular understanding in less than a decade. Nat Rev Mol Cell Biol 8: 931-937, 2007.

4. Galluzzi L, Pietrocola F, Bravo-San Pedro JM, Amaravadi RK, Baehrecke EH, Cecconi F, Codogno P, Debnath J, Gewirtz DA, Karantza V, et al: Autophagy in malignant transformation and cancer progression. EMBO J 34: 856-880, 2015.

5. Amaravadi R, Kimmelman AC and White E: Recent insights into the function of autophagy in cancer. Genes Dev 30: 1913-1930, 2016.

6. Levy JM and Thorburn A: Targeting autophagy during cancer therapy to improve clinical outcomes. Pharmacol Ther 131: 130-141, 2011.

7. Towers CG and Thorburn A: Therapeutic targeting of autophagy. EBioMedicine 14: 15-23, 2016.

8. Jing Z, Han W, Sui X, Xie J and Pan H: Interaction of autophagy with microRNAs and their potential therapeutic implications in human cancers. Cancer Lett 356B: 332-338, 2015.

9. Fu LL, Wen X, Bao JK and Liu B: MicroRNA-modulated autophagic signaling networks in cancer. Int J Biochem Cell Biol 44: 733-736, 2012.

10. Xu J, Wang Y, Tan X and Jing H: MicroRNAs in autophagy and their emerging roles in crosstalk with apoptosis. Autophagy 8: 873-882, 2012.

11. Iorio MV and Croce CM: MicroRNAs in cancer: Small molecules with a huge impact. J Clin Oncol 27: 5848-5856, 2009.

12. Bartel DP: MicroRNAs: Genomics, biogenesis, mechanism, and function. Cell 116: 281-297, 2004.

13. Huang Y, Chuang AY and Ratovitski EA: Phospho- $\Delta$ Np63 $\alpha /$ miR-885-3p axis in tumor cell life and cell death upon cisplatin exposure. Cell Cycle 10: 3938-3947, 2011.

14. Zhu H, Wu H, Liu X, Li B, Chen Y, Ren X, Liu CG and Yang JM: Regulation of autophagy by a Beclin 1-targeted microRNA, miR-30a, in cancer cells. Autophagy 5: 816-823, 2009.

15. Chang Y, Yan W, He X, Zhang L, Li C, Huang H, Nace G, Geller DA, Lin J and Tsung A: miR-375 inhibits autophagy and reduces viability of hepatocellular carcinoma cells under hypoxic conditions. Gastroenterology 143: 177-187.e8, 2012.

16. Korkmaz G, le Sage C, Tekirdag KA, Agami R and Gozuacik D: miR-376b controls starvation and mTOR inhibition-related autophagy by targeting ATG4C and BECN1. Autophagy 8: 165-176, 2012.

17. Tekirdag KA, Korkmaz G, Ozturk DG, Agami R and Gozuacik D: MIR181A regulates starvation- and rapamycininduced autophagy through targeting of ATG5. Autophagy 9: 374-385, 2013.

18. Ren Y, Chen Y, Liang X, Lu Y, Pan W and Yang M: miRNA-638 promotes autophagy and malignant phenotypes of cancer cells via directly suppressing DACT3. Cancer Lett 390: 126-136, 2017.

19. Guo W, Wang H, Yang Y, Guo S, Zhang W, Liu Y, Yi X, Ma J, Zhao T, Liu L, et al: Down-regulated miR-23a contributes to the metastasis of cutaneous melanoma by promoting autophagy. Theranostics 7: 2231-2249, 2017.

20. Wang Y, Zhang X, Tang W, Lin Z, Xu L, Dong R, Li Y, Li J, Zhang Z, Li X, et al: miR-130a upregulates mTOR pathway by targeting TSC1 and is transactivated by NF- $\kappa \mathrm{B}$ in high-grade serous ovarian carcinoma. Cell Death Differ 24: 2089-2100, 2017. 
21. Du X, Huo X, Yang Y, Hu Z, Botchway BOA, Jiang Y and Fang M: miR-124 downregulates BACE 1 and alters autophagy in APP/PS1 transgenic mice. Toxicol Lett 280: 195-205, 2017.

22. Triozzi PL, Achberger S, Aldrich W, Crabb JW, Y and Singh AD: Association of tumor and plasma microRNA expression with tumor monosomy-3 in patients with uveal melanoma. Clin Epigenetics 8: 80, 2016.

23. Zhou Z, Wang Z, Wei H, Wu S, Wang X and Xiao J: Promotion of tumour proliferation, migration and invasion by miR-92b in targeting RECK in osteosarcoma. Clin Sci (Lond) 130: 921-930, 2016.

24. Huang J, Wang B, Hui K, Zeng J, Fan J, Wang X, Hsieh JT, He D and Wu K: miR-92b targets DAB2IP to promote EMT in bladder cancer migration and invasion. Oncol Rep 36: 1693-1701, 2016.

25. Zhao C, Zhao F, Feng H, Xu S and Qin G: MicroRNA-92b inhibits epithelial-mesenchymal transition-induced migration and invasion by targeting Smad3 in nasopharyngeal cancer. Oncotarget 8: 91603-91613, 2017.

26. Livak KJ and Schmittgen TD: Analysis of relative gene expression data using real-time quantitative PCR and the 2(-Delta Delta C(T)) Method. Methods 25: 402-408, 2001.

27. Nowakowski TJ, Fotaki V, Pollock A, Sun T, Pratt T and Price DJ: MicroRNA-92b regulates the development of intermediate cortical progenitors in embryonic mouse brain. Proc Natl Acad Sci USA 110: 7056-7061, 2013.

28. Zhou X, Zhu W, Li H, Wen W, Cheng W, Wang F, Wu Y, Qi L, Fan Y, Chen Y, et al: Diagnostic value of a plasma microRNA signature in gastric cancer: A microRNA expression analysis. Sci Rep 5: 11251, 2015.

29. Wu ZB, Cai L, Lin SJ, Lu JL, Yao Y and Zhou LF: The miR-92b functions as a potential oncogene by targeting on Smad3 in glioblastomas. Brain Res 1529: 16-25, 2013.

30. Li Y, Li L, Guan Y, Liu X, Meng Q and Guo Q: miR-92b regulates the cell growth, cisplatin chemosensitivity of A549 non smal cell lung cancer cell line and target PTEN. Biochem Biophys Res Commun 440: 604-610, 2013.

31. Ma G, Jing C, Li L, Huang F, Ding F, Wang B, Lin D, Luo A and Liu Z: MicroRNA-92b represses invasion-metastasis cascade of esophageal squamous cell carcinoma. Oncotarget 7 : 20209-20222, 2016 .

32. Ma G, Jing C, Huang F, Li X, Cao X and Liu Z: Integrin $\alpha 6$ promotes esophageal cancer metastasis and is targeted by miR-92b. Oncotarget 8: 6681-6690, 2017.

33. Vidigal JA and Ventura A: The biological functions of miRNAs: Lessons from in vivo studies. Trends Cell Biol 25: 137-147, 2015.

34. Müller J, Hart CM, Francis NJ, Vargas ML, Sengupta A, Wild B, Miller EL, O'Connor MB, Kingston RE and Simon JA: Histone methyltransferase activity of a Drosophila Polycomb group repressor complex. Cell 111: 197-208, 2002.
35. Czermin B, Melfi R, McCabe D, Seitz V, Imhof A and Pirrotta V: Drosophila enhancer of Zeste/ESC complexes have a histone H3 methyltransferase activity that marks chromosomal Polycomb sites. Cell 111: 185-196, 2002

36. Varambally S, Dhanasekaran SM, Zhou M, Barrette TR, Kumar-Sinha C, Sanda MG, Ghosh D, Pienta KJ, Sewalt RG, Otte AP, et al: The polycomb group protein EZH2 is involved in progression of prostate cancer. Nature 419: 624-629, 2002.

37. Kleer CG, Cao Q, Varambally S, Shen R, Ota I, Tomlins SA, Ghosh D, Sewalt RG, Otte AP, Hayes DF, et al: EZH2 is a marker of aggressive breast cancer and promotes neoplastic transformation of breast epithelial cells. Proc Natl Acad Sci USA 100: 11606-11611, 2003

38. Collett K, Eide GE, Arnes J,, Stefansson IM, Eide J, Braaten A, Aas T, Otte AP and Akslen LA: Expression of enhancer of zeste homologue 2 is significantly associated with increased tumor cell proliferation and is a marker of aggressive breast cancer. Clin Cancer Res 12: 1168-1174, 2006

39. Liu F, Gu L, Cao Y, Fan X, Zhang F and Sang M: Aberrant overexpression of EZH2 and $\mathrm{H} 3 \mathrm{~K} 27 \mathrm{me} 3$ serves as poor prognostic biomarker for esophageal squamous cell carcinoma patients. Biomarkers 21: 80-90, 2016.

40. Wei FZ, Cao Z, Wang X, Wang H, Cai MY, Li T, Hattori N, Wang D, Du Y, Song B, et al: Epigenetic regulation of autophagy by the methyltransferase EZH2 through an MTOR-dependent pathway. Autophagy 11: 2309-2322, 2015.

41. Schlesinger Y, Straussman R, Keshet I, Farkash S, Hecht M, Zimmerman J,Eden E, YakhiniZ, Ben-Shushan E, Reubinoff BE, et al: Polycomb-mediated methylation on Lys27 of histone $\mathrm{H} 3$ pre-marks genes for de novo methylation in cancer. Nat Genet 39: 232-236, 2007.

42. Tonini T, D'Andrilli G, Fucito A, Gaspa L and Bagella L: Importance of Ezh2 polycomb protein in tumorigenesis process interfering with the pathway of growth suppressive key elements. J Cell Physiol 214: 295-300, 2008.

43. Widschwendter M, Fiegl H, Egle D, Mueller-Holzner E, Spizzo G, Marth C, Weisenberger DJ, Campan M, Young J, Jacobs I, et al: Epigenetic stem cell signature in cancer. Nat Genet 39: 157-158, 2007.

This work is licensed under a Creative Common Attribution-NonCommercial-NoDerivatives 4.0 International (CC BY-NC-ND 4.0) License. 